\title{
Dust flow in gas disks in the presence of embedded planets
}

\author{
S.-J. Paardekooper ${ }^{1}$ and G. Mellema ${ }^{2,1}$ \\ 1 Leiden Observatory, Postbus 9513, 2300 RA Leiden, The Netherlands \\ e-mail: paardeko@strw.leidenuniv.nl \\ 2 ASTRON, Postbus 2, 7990 AA Dwingeloo, The Netherlands \\ e-mail: gmellema@astron.nl
}

Received 31 October 2005 / Accepted 21 February 2006

ABSTRACT

\begin{abstract}
Aims. We study the dynamics of gas and dust in a protoplanetary disk in the presence of embedded planets. We investigate the conditions for dust-gap formation in terms of particle size and planetary mass. We also monitor the amount of dust that is accreted by the planet relative to the amount of gas, which is an important parameter in determining the enrichment of solids in giant planets compared to the solid content of the central star.

Methods. We use a new two-fluid hydrodynamics code to solve the flow equations for both gas and dust. For the gas, we use a Godunov-type scheme with an approximate Riemann solver (the Roe solver). The dust is treated as a pressureless fluid by essentially the same numerical method as is used for the gas.

Results. We find that it only takes a planet of 0.05 Jupiter masses to open up a gap in a disk with a significant population of mm-sized particles. Dust particles larger than $150 \mu \mathrm{m}$ participate in gap formation. We also find that the formation of the gap severely slows down dust accretion compared to that in the gas. Therefore, it is not possible to enrich a newly formed giant planet in solids, if these solids are contained in particles with sizes from $150 \mu \mathrm{m}$ to approximately $10 \mathrm{~cm}$.
\end{abstract}

Key words. hydrodynamics - methods: numerical - stars: planetary systems: protoplanetary disks

\section{Introduction}

Dust plays a major role in planet formation, theoretically as well as observationally. First of all, terrestial planets consist mainly of solid material. They are giant agglomerates of interstellar dust particles, and therefore the behaviour of these small particles is important during the first stages of planet formation. Whether they grow to kilometer-sized bodies by slow coagulation or through the gravitational instability of a thin dust layer in the midplane of the disk (Goldreich \& Ward 1973) is still an open question. A mixture of equal amounts of gas and dust is subject to streaming instabilities (Youdin \& Goodman 2005), and it is not clear whether the resulting turbulent structure will prohibit gravitational instabilities or enhance them (Johansen et al. 2006). To answer this question, multi-component simulations are needed in which gas and dust are able to evolve separately.

Secondly, giant planets can be formed either by gas accretion onto a solid core (Pollack et al. 1996) or by direct gravitational fragmentation of the disk (Boss 1997). In the core accretion model, dust growth is of critical importance to build up a massive core before the gas disk dissipates. The gravitational instability model does not rely on the existence of such a core, but still, accretion of dust in a later stage will determine the enrichment in solids with respect to the solar nebula, which is an important observational constraint (Guillot et al. 2004).

Finally, dust is the major source of continuum radiation in the disk. Therefore, infra-red and sub-millimeter emissions mainly indicate the density and temperature of the dust disk. The relation of the dust to the density of the gas disk can be quite complicated if the disk has a non-trivial structure, especially if the dust particles are only marginally coupled to the gas.
It is therefore very important to study dust dynamics in gas disks. Axisymmetric disks can be handled analytically, and various authors have studied dust migration in these disks (Weidenschilling 1977; Takeuchi \& Lin 2002). Particles move both inward and outward under the influence of gas drag, depending on their distance from the midplane, but the average migration is inward (Takeuchi \& Lin 2002). Haghighipour \& Boss (2003) studied dust migration in a non-uniform axisymmetric nebula, and Takeuchi \& Artymowicz (2001) investigated the effect of a sharp disk edge on dust migration. The overall conclusion is that the structure of the gas disk can have a significant effect on the dust distribution, and that the distribution of gas and dust can differ substantially.

When the gas distribution becomes more complicated, one has to rely on numerical simulations. Suttner \& Yorke (2001) studied dust evolution during the initial collapse of the nebula with a two-dimensional multi-fluid hydrodynamics code to obtain the large-scale dust distribution. Johansen \& Klahr (2005) studied the effect of MHD turbulence on the dust distribution. These multi-fluid calculations are computationally very expensive, requiring more than twice the computing time for ordinary gas-dynamical simulations, even if one solves for only a single dust particle size.

To compare dust observations with gas-dynamical simulations, but at the same time avoid the large computational effort of these multi-fluid calculations, one can assume a fixed dust-togas ratio and solve only for the gas dynamics only. The effects of an embedded planet on a gaseous disk have been studied numerically before by multiple authors (e.g. Kley 1999; Nelson et al. 2000; Lubow et al. 1999; D'Angelo et al. 2002). Wolf et al. (2002) used these results to simulate observations of gaps in 
protoplanetary disks. Their calculations are valid only for the smallest particles that couple extremely well to the gas.

However, in a protoplanetary disk where grain growth is very important (see, for example, Dullemond \& Dominik 2005), a significant fraction of the dust population is large enough to be only marginally coupled to the gas. Paardekooper \& Mellema (2004) showed that including the full gas and dust dynamics leads to a dramatic evolution of the dust component in the disk. Planets not massive enough to open up a gap in the gas disk do so in the dust disk, which should make the orbit of even $0.05 M_{\mathrm{J}}$ planets visible to ALMA (the Atacama Large Millimeter Array).

In this paper we continue our investigation of dust flow in gas disks around embedded planets, with more attention to detail than in Paardekooper \& Mellema (2004). In Sect. 2, we review the equations governing dust flow and gas-dust interaction. Section 3 is devoted to the numerical method, and we discuss the initial and boundary conditions in Sect. 4. In Sect. 5, we present the results. Section 6 is reserved for a short discussion, and we conclude in Sect. 7.

\section{Basic equations}

Protoplanetary disks are fairly thin, i.e. the vertical thickness $H$ is small compared with the distance $r$ from the centre of the disk. Typically we use $H / r=h=0.05$. It is therefore convenient to average the equations of motion vertically and to work with vertically averaged quantities only.

The governing equations are then solved in a cylindrical coordinate frame $(r, \phi)$, centred on the central star and corotating with the embedded planet that has a Keplerian angular velocity $\Omega$.

\subsection{Gas}

The equations that control the gas evolution are described in detail in Paardekooper \& Mellema (2005). We do not solve the energy equation, but instead use an isothermal equation of state:

$P=c_{\mathrm{s}}^{2} \Sigma_{\mathrm{g}}$,

where $\Sigma_{\mathrm{g}}$ is the surface density of the gas and $P$ is the vertically averaged pressure.

The isothermal sound speed $c_{\mathrm{s}}$ is, in hydrostatic equilibrium, directly related to the disk thickness:

$c_{\mathrm{s}}=h v_{\mathrm{K}}$,

where $v_{\mathrm{K}}$ is the Keplerian velocity.

We use a constant kinematic viscosity $v$, which is set by assuming an $\alpha$-parameter (Shakura \& Sunyaev 1973) of 0.004 at the location of the planet:

$v=\left.\alpha c_{\mathrm{s}} H\right|_{r=1}$.

\subsection{Dust}

We treat the dust as a pressureless fluid; its evolution is governed by conservation of mass and, in absence of external sources, conservation of radial and angular momentum. These can be written in the following compact form:

$\frac{\partial \boldsymbol{W}}{\partial t}+\frac{\partial \boldsymbol{F}}{\partial r}+\frac{\partial \boldsymbol{G}}{\partial \phi}=\boldsymbol{S}$ where $\boldsymbol{W}$ is called the state vector and where $\boldsymbol{F}$ and $\boldsymbol{G}$ are the fluxes in the radial and azimuthal direction, respectively. $S$ is called the source term.

The components of the state, fluxes, and source-term vectors can be written in the following form:

$\boldsymbol{W}=r\left(\Sigma, \Sigma v_{r}, \Sigma v_{\phi}\right)$
$\boldsymbol{F}=r\left(\Sigma v_{r}, \Sigma v_{r}^{2}, \Sigma v_{r} v_{\phi}\right)$
$\boldsymbol{G}=r\left(\Sigma v_{\phi}, \Sigma v_{r} v_{\phi}, \Sigma v_{\phi}^{2}\right)$

$\boldsymbol{S}=\left(\begin{array}{c}0 \\ \Sigma r^{2}\left(v_{\phi}+\Omega\right)^{2}-\Sigma r \frac{\partial \Phi}{\partial r}+r f_{\mathrm{d}, r} \\ -2 \Sigma v_{r}\left(\Omega+v_{\phi}\right)-\frac{\Sigma}{r} \frac{\partial \Phi}{\partial \phi}+r f_{\mathrm{d}, \phi}\end{array}\right)$

Here, $\Sigma$ denotes the dust surface density, $v_{r}$ and $v_{\phi}$ the radial and angular velocity, respectively, $\Omega$ the angular velocity of the coordinate frame (corotating with the planet), and $\Phi$ the gravitational potential of the central star and the planet. The gravitational potential of the planet is softened over a fraction 0.2 of the Roche lobe of the planet. The potential also contains terms due to the non-inertial nature of the coordinate system. The drag forces are incorporated through $f_{\mathrm{d}, r}$ and $f_{\mathrm{d}, \phi}$.

The major difference between the equations for the gas and the dust is the absence of pressure in the latter. In this sense, the dust fluid behaves like a gas that is always moving with supersonic velocity. This implies that near shock waves, where the gas goes from sonic to supersonic flow and where large density and velocity gradients are present, dust and gas behave in very different ways.

The basic building block for Godunov-type hydrodynamic solvers is the Riemann problem, consisting of two stationary states separated by a discontinuity. If we consider the two states as belonging to neighbouring grid cells, with the discontinuity at the interface between the cells, the analytic solution of the Riemann problem can be used to define an interface flux between the cells. Popular schemes of this type are the Piecewise Parabolic Method (PPM, Woodward \& Colella 1984) and the Roe solver (Roe 1981).

For pressureless fluids, the solution to the Riemann problem differs significantly from the case with pressure. Figure 1 shows the analytic solutions for the gas (dots) as well as the dust density (solid line), with initial conditions $\rho_{\mathrm{L}}=1.0, \rho_{\mathrm{R}}=0.5, u_{\mathrm{L}}=0.5$, and $u_{\mathrm{R}}=0.25$. The gas solution consists of two waves moving at speeds $\hat{u}-c_{\mathrm{s}}$ and $\hat{u}+c_{\mathrm{s}}$, where

$\hat{u}=\frac{\sqrt{\rho_{\mathrm{L}}} u_{\mathrm{L}}+\sqrt{\rho_{\mathrm{R}}} u_{\mathrm{R}}}{\sqrt{\rho_{\mathrm{L}}}+\sqrt{\rho_{\mathrm{R}}}}$.

If we now let $c_{\mathrm{s}} \rightarrow 0$ to obtain a pressureless fluid, the separation between the waves becomes smaller and smaller, until they overlap at $c_{\mathrm{s}}=0$. The overlapping waves create a delta-function singularity that moves with velocity $\hat{u}$ (e.g. Leveque 2004). The singularity arises because Eq. (4) is only weakly hyperbolic, in contrast with the gas hydrodynamical equations, which are strictly hyperbolic.

Figure 1 deals with the case $u_{\mathrm{L}}>u_{\mathrm{R}}$. When $u_{\mathrm{L}}<u_{\mathrm{R}}$, the solution consists of the two states moving away from each other with a vacuum in between. Note that for both cases, the flux is well defined everywhere but at the position of the delta function.

The solution to the Riemann problem not only forms the basis for our numerical method (see Sect. 3), but from Fig. 1 it is also clear that gas and dust react very differently to physical shocks, which may lead to gas-dust separation when shock waves are present in the computational domain. 


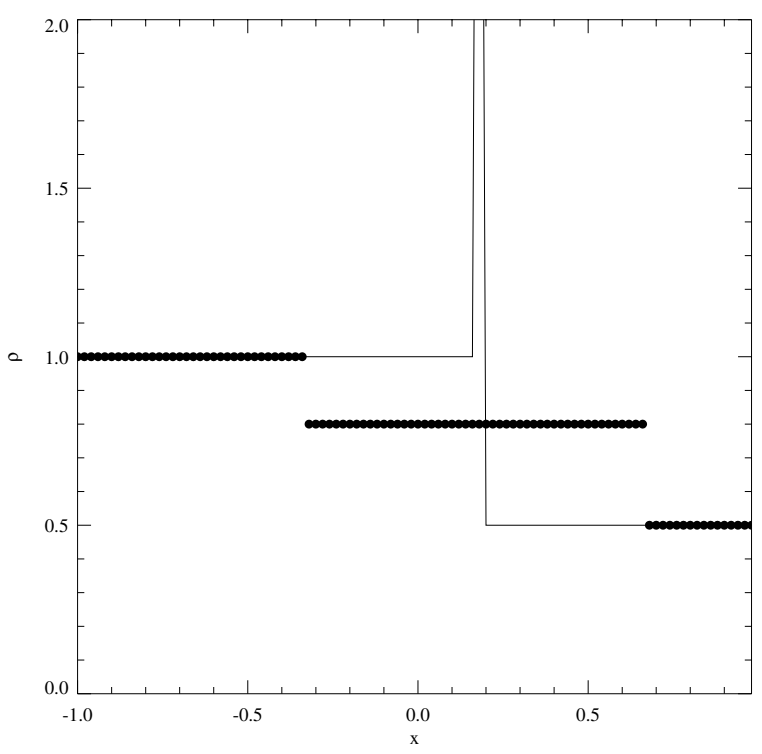

Fig. 1. Solution to the Riemann problem with initial conditions $\rho_{\mathrm{L}}=$ $1.0, \rho_{\mathrm{R}}=0.5, u_{\mathrm{L}}=0.5$, and $u_{\mathrm{R}}=0.25$. The solid line represents the dust density and the dots represent the gas density, both at $t=0.5$. For the gas, the speed of sound was set to 1.0.

\subsection{Gas-dust interaction}

The interaction between the gas fluid and the dust fluid occurs only through the drag forces. The nature of the drag force depends on the size of the particles with respect to the mean free path of the gas molecules. We consider only spherical particles with radius $s$. Because the main constituent of the gas is molecular hydrogen, for the mean free path we can write:

$\lambda=\frac{m_{\mathrm{H}_{2}}}{\pi \rho_{\mathrm{g}} r_{\mathrm{H}_{2}}{ }^{2}}$.

When the particle size is small, compared to $\lambda$, we are in the Epstein regime, whereas $s \gg \lambda$ corresponds to the Stokes regime. For particles that move subsonically through the gas, the transition occurs around $\lambda / s=4 / 9$. For typical midplane densities, particles larger than approximately $50 \mathrm{~cm}$ move in the Stokes regime. In this paper we will focus on particles smaller than $\sim 1 \mathrm{~cm}$, and therefore we can safely use the Epstein drag law.

In the Epstein regime, when the relative velocity of gas and dust is much smaller than the local sound speed, the drag force is written as:

$f_{\mathrm{d}}=-\Sigma \frac{\Omega_{\mathrm{K}}}{T_{\mathrm{s}}} \Delta \boldsymbol{v}$

where $\Omega_{\mathrm{K}}$ is the Keplerian angular velocity, $\Delta \boldsymbol{v}$ is the velocity difference between gas and dust, and $T_{\mathrm{s}}$ is the dimensionless stopping time (Takeuchi \& Lin 2002):

$T_{\mathrm{s}}=\sqrt{\frac{\pi}{8}} \frac{\rho_{\mathrm{p}} s v_{\mathrm{K}}}{\rho_{\mathrm{g}} r c_{\mathrm{s}}}$.

Here, $\rho_{\mathrm{p}}$ is the particle internal density, $s$ is the radius of the particle, and $c_{\mathrm{s}}$ is the sound speed. We have used $\rho_{\mathrm{p}}=1.25 \mathrm{~g} \mathrm{~cm}^{-3}$, and the isothermal sound speed is given by Eq. (2). The gas density $\rho_{\mathrm{g}}$ is found by using $\rho_{\mathrm{g}}=\Sigma_{\mathrm{g}} / 2 H$.

For particles that move very supersonically through the gas, the drag force becomes:

$f_{\mathrm{d}}=-\Sigma \frac{3}{4} \sqrt{\frac{\pi}{8}} \frac{\Omega_{\mathrm{K}}}{T_{\mathrm{s}}} \frac{|\Delta \boldsymbol{v}|}{c_{\mathrm{s}}} \Delta \boldsymbol{v}$.
A standard way to connect the supersonic to the subsonic regime is (Kwok 1975):

$f_{\mathrm{d}}=-\Sigma \frac{\Omega_{\mathrm{K}}}{T_{\mathrm{s}}} \sqrt{1+\frac{9 \pi}{128} \frac{|\Delta \boldsymbol{v}|^{2}}{c_{\mathrm{s}}^{2}}} \Delta \boldsymbol{v}$.

See Woitke \& Helling (2003) for the general case.

Only very massive planets of approximately $1 M_{\mathrm{J}}$ are able to accelerate the larger particles to velocities that are comparable to the speed of sound. For the low-mass planets considered in Paardekooper \& Mellema (2004), the subsonic version of the drag force proved to be sufficient.

Particles with $T_{\mathrm{s}} \ll 1$ are well-coupled to the gas, and the radial drift velocity can be written as (Takeuchi \& Lin 2002):

$v_{r, \text { drift }}=-\eta T_{\mathrm{s}} v_{\mathrm{K}}$

where $\eta$ is the ratio of the gas pressure gradient to the stellar gravity in the radial direction:

$\eta=-h^{2} \frac{\partial \log P}{\partial \log r}=h^{2}\left(1-\frac{\partial \log \Sigma_{\mathrm{g}}}{\partial \log r}\right)$.

The direction in which the dust moves with respect to the gas is determined by the sign of $\eta$, and in general the pressure gradient is negative, making $\eta$ positive, which leads to an inward migration of the dust particles. However, if there is a large positive density gradient $\left(\partial \log \Sigma_{\mathrm{g}} / \partial \log r>1\right.$, see Eq. (16)), as there is near the outer edge of a density gap, the dust particles can move outward. On the other hand, if there is an extra large negative density gradient, as is the case near the inner edge of a density gap, the dust moves inward with higher velocity.

Thus, we expect that near the gap edges, the larger particles decouple from the gas, altering the dust-to-gas ratio in that region. Furthermore, the drag forces may not be large enough to prevent gas-dust separation by shocks. Both processes should play a role in the dust dynamics near the embedded planet.

\section{Numerical method}

\subsection{Gas and dust advection}

For the evolution of the gas component, we use the RODEO method described by Paardekooper \& Mellema (2005). It is a second-order Eulerian hydrodynamics code, which uses an approximate Riemann solver (Roe 1981). It derives from a general relativistic method (Eulderink \& Mellema 1995), and it is especially suitable to treat non-Cartesian, non-inertial coordinate systems. A module for Adaptive Mesh Refinement (AMR) can be used to obtain high resolution near the planet.

For the dust, we use the method outlined in Leveque (2004), which has the advantage of being similar to the method used for the gas. Below, we only consider the radial direction. The azimuthal direction is handled in the same way.

For every cell interface, we consider the Riemann problem defined by a left state $\boldsymbol{W}_{\mathrm{L}}$ and a right state $\boldsymbol{W}_{\mathrm{R}}$ with corresponding fluxes. When $v_{r, \mathrm{~L}}<0<v_{r, \mathrm{R}}$, the solution to the Riemann problem gives us a vacuum state at the cell interface, so we set the interface flux $\boldsymbol{F}_{\text {int }}=\mathbf{0}$. Otherwise, we compute the shock speed $\hat{v}$ from Eq. (9), and compute the interface flux according to

$$
\boldsymbol{F}_{\text {int }}= \begin{cases}\boldsymbol{F}_{\mathrm{L}} & \text { if } \hat{v}>0 \\ \left(\boldsymbol{F}_{\mathrm{L}}+\boldsymbol{F}_{\mathrm{R}}\right) / 2 & \text { if } \hat{v}=0 \\ \boldsymbol{F}_{\mathrm{R}} & \text { if } \hat{v}<0\end{cases}
$$


In the first and the last case, the cell interface is respectively left and right of the delta shock, allowing for an easy definition of the interface flux. When $\hat{v}=0$, however, the delta shock is exactly at the cell interface. We then choose to distribute this shock equally over both cells.

To put this in the language of Paardekooper \& Mellema (2005), we write $\boldsymbol{F}=\mathcal{A} \boldsymbol{W}$, where the matrix $\mathcal{A}$ is given by:

$\mathcal{A}=\left(\begin{array}{ccc}v_{r} & 0 & 0 \\ 0 & v_{r} & 0 \\ 0 & 0 & v_{r}\end{array}\right)$.

The eigenvalues of this matrix are:

$\lambda_{1}=\lambda_{2}=\lambda_{3}=v_{r}$

The corresponding eigenvectors read:

$\boldsymbol{e}_{1}=(1,0,0)$

$\boldsymbol{e}_{2}=(0,1,0)$

$\boldsymbol{e}_{3}=(0,0,1)$.

A vector $\boldsymbol{\Delta} \equiv\left(\Delta_{\rho}, \Delta_{r}, \Delta_{\phi}\right)$ can be projected on these eigenvectors using the following projection coefficients, found by solving the system $\boldsymbol{C b}=\boldsymbol{\Delta}$, where $C$ is the matrix with the eigenvectors:

$b_{1}=\Delta_{\rho}$

$b_{2}=\Delta_{r}$

$b_{3}=\Delta_{\phi}$.

To approximate the matrix $\mathcal{A}$ at an interface of two grid cells, we use Roe-averages. If we then project the flux difference across the interface we are considering onto the eigenvectors of $\mathcal{A}$ :

$\boldsymbol{F}_{\mathrm{R}}-\boldsymbol{F}_{\mathrm{L}}=\sum b_{\mathrm{k}} \boldsymbol{e}_{\mathrm{k}}$

we can then define the first order interface flux just as it was defined in the gas case:

$\boldsymbol{F}_{\text {int }}=\frac{1}{2}\left(\boldsymbol{F}_{\mathrm{L}}+\boldsymbol{F}_{\mathrm{R}}\right)-\frac{1}{2} \sum \sigma_{\mathrm{k}} b_{\mathrm{k}} \boldsymbol{e}_{\mathrm{k}}$,

where $\sigma_{\mathrm{k}}=\operatorname{sign}\left(\lambda_{\mathrm{k}}\right)$. When $\lambda_{\mathrm{k}}=0$, we take $\sigma_{\mathrm{k}}=0$. It is easy to show that this version of the interface flux corresponds exactly with Eq. (17). Having the interface flux in this form, we can immediately write down a second-order interface flux that is again analogous to the gas case:

$\boldsymbol{F}_{\text {int }}=\frac{1}{2}\left(\boldsymbol{F}_{\mathrm{L}}+\boldsymbol{F}_{\mathrm{R}}\right)-\frac{1}{2} \sum\left(\sigma_{\mathrm{k}} a_{\mathrm{k}}-\left(\sigma_{\mathrm{k}}-v_{\mathrm{k}}\right) \psi_{\mathrm{k}}\right) \lambda_{\mathrm{k}} \boldsymbol{e}_{\mathrm{k}}$,

where $v_{\mathrm{k}}=\lambda_{\mathrm{k}} \Delta t / \Delta r$ and $\psi$ is the flux limiter. We have used the same limiter as in Paardekooper \& Mellema (2005) for both gas and dust in all simulations.

\subsection{Accretion}

We model accretion onto the planet by taking mass away from the grid at the location of the planet. There are two parameters governing the amount of mass taken away at each time-step: the accretion area and the accretion rate. We take the accretion area to be a circle with a radius equal to a tenth of the Roche lobe of the planet, and the accretion rate to be such that the time scale for emptying this region is equal to three fifths of the orbital time scale of the planet. The same parameters were used in D'Angelo et al. (2002) and Paardekooper \& Mellema (2005).

\subsection{Source terms}

All the source terms except the drag forces are integrated using stationary extrapolation (Paardekooper \& Mellema 2005). The combination of exact extrapolation for the angular momentum and approximate extrapolation for the other geometrical source terms was shown to give reliable results for single-fluid calculations of the planet-disk problem. Because these results were not affected by increasing the resolution, we can conclude that using this treatment of the source terms is accurate enough. However, when other forces besides gravity are included, it is not guaranteed that a stationary solution exists. Simis et al. (2001) found that the source terms arising in two-fluid calculations are best integrated analytically. Therefore, the drag forces are incorporated separately using an ordinary differential equation:

$\frac{\mathrm{d}}{\mathrm{d} t}(\Sigma \boldsymbol{v})=\boldsymbol{f}_{\mathrm{d}}$.

During this step, we keep the dust and gas density fixed. Using Eq. (14), we can write an ordinary differential equation for the velocity difference between gas and dust:

$\frac{\mathrm{d}}{\mathrm{d} t}(\Delta v)=-\alpha \sqrt{1+\beta \Delta v^{2}} \Delta v$

where $\alpha=\frac{\Omega_{\mathrm{K}}}{T_{\mathrm{s}}}$ and $\beta=\frac{9 \pi}{128 c_{\mathrm{s}}^{2}}$. This equation can be integrated analytically to give

$\Delta \boldsymbol{v}(t)=\frac{2 \Delta v_{0} \exp (-\alpha t)}{1+\sqrt{1+\beta \Delta v_{0}^{2}}-\frac{\beta v_{0}^{2} \exp (-2 \alpha t)}{1+\sqrt{1+\beta \Delta v_{0}^{2}}}}$.

Note that when $\beta=0$, we recover the ordinary exponential decay of the velocity difference. Using conservation of total momentum:

$\Sigma \boldsymbol{v}+\Sigma_{g} \boldsymbol{v}_{g}=$ constant

we can solve for the evolution of the velocities of gas and dust separately, while keeping the total momentum conserved exactly.

\subsection{Test problem}

Gasdynamical codes are often tested against a simple Riemann problem (Sod 1978), also known as the shock tube. In this section, we test the method for dust advection against the Riemann problem shown in Fig. 1, using a 100-zone grid. We also study the effect of the interaction between gas and dust. The initial dust-to-gas mass ratio is 1 . The simulations were run until $t=0.5$, which took approximately 50 time-steps.

In Fig. 2, we show the results for three different stopping times: $T_{\mathrm{s}}=\infty$ (left panels), $T_{\mathrm{s}}=0.5$ (middle panels), and $T_{\mathrm{s}}=$ 0.05 (right panels). We see that for the case of no interaction, we reproduce the result of Fig. 1 for gas and dust. The delta shock is smeared over approximately 4 computational cells, but it moves at the correct speed. Note that because the velocity is less than the sound speed everywhere, the waves in the gas are not shock waves, but are simply sound waves.

For the middle panels, the stopping time is comparable to the sound-crossing time and the dust velocity increases towards the gas velocity, while by conservation of momentum, the gas velocity decreases with respect to the left panels. The velocities approach each other the fastest at the position of the delta shock which has decreased in amplitude because the gas and dust densities also approach each other. 


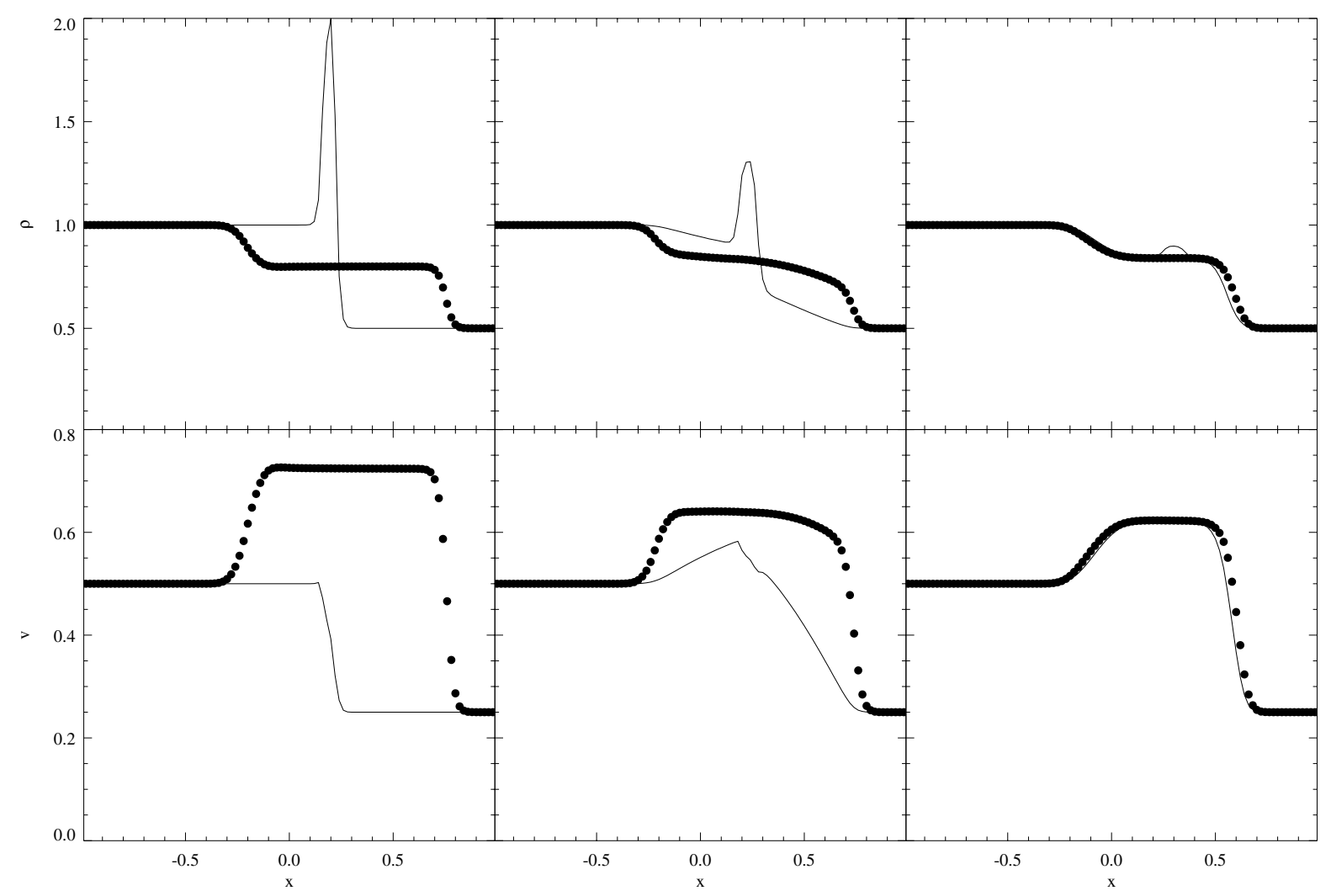

Fig. 2. Numerical results for the Riemann problem of Fig. 1 for gas (dots) and dust (solid line). The top row shows the density, the bottom row the velocity. Left panels: no gas-dust interaction. Middle panels: $T_{\mathrm{s}}=0.5$. Right panels: $T_{\mathrm{s}}=0.05$.

When we decrease the stopping time by a factor of ten, the densities and velocities of gas and dust are almost equal everywhere. In the right panels of Fig. 2, there is only a small bump left of the delta shock, and the gas has lost enough momentum to the dust to visibly slow down the sound waves. Note that this only happens because the gas and dust densities were equal initially. For an interstellar dust-to-gas ratio of 1:100, the gas is not affected by the dust.

\subsection{Limits}

The approach presented here cannot be used in all circumstances. To begin with, the continuum form for the equations of motion is only valid if two requirements are met. First, there need to be enough particles in the volume under consideration (in our case, one grid cell) to work with average states. Second, there need to be enough interparticle collisions to make such an average state meaningful. For a dust fluid, both criteria are only fullfilled if the particles are well-coupled to the gas (Garaud et al. 2004). In other words, we can only work with particles for which $T_{\mathrm{s}} \ll 1$ everywhere in the computational domain. For larger dust boulders, a particle-based method is more appropriate (Johansen et al. 2005; Fromang \& Nelson 2005).

On the other hand, when the stopping time is very small, the source terms due to gas-dust friction become stiff. Even though we integrate these source terms in an implicit way, which makes the method stable for these small particles, it does not necessarily give us the correct answer. This is because the gas source terms due to pressure are dealt with in the advection step, and pressure is precisely what causes gas-dust separation. Therefore, even for $T_{\mathrm{s}}=0$, the dust would numerically slowly drift inward, while according to Eq. (15), its radial velocity should be zero.
The range in $T_{\mathrm{s}}$ that we can consider is therefore also bounded from below. The time scale for the advection (the hydrodynamical time-step, determined by the Courant-Friedrichs-Lewy (CFL) condition) should not be much larger than the time scale for gas-dust coupling. Combining the two limits, we can conclude that we can only consider particles for which the stopping time satisfies:

$\Omega_{\mathrm{K}} \Delta t \approx T_{\mathrm{S}} \ll 1$.

In practice, for the gas densities considered here (see Sect. 4), Eq. (29) limits the particle size from below at approximately $150 \mu \mathrm{m}$ and from above at approximately $1 \mathrm{~cm}$. In all our simulations we checked that lowering the time-step $\Delta t$ did not influence the results.

It is important to realize that Eq. (29) should hold true everywhere on the grid. When the gas density changes significantly, for example due to the formation of a gap, the stopping time can vary by orders of magnitude. The condition $T_{\mathrm{s}} \ll 1$ for gapopening planets makes the simulations very expensive. To do the calculations in a reasonable amount of time, we restrict our parameter space to non-gap-opening planets. Note, however, that gap-opening planets also pose problems for particle-based methods because very many particles are needed to achieve enough resolution inside the gap.

The two-dimensional nature of these simulations also puts conditions on the size of the parameter space we can consider. When the Roche lobe of the planet is smaller than the disk thickness, three-dimensional effects come into play, lowering accretion and migration rates for example (D'Angelo et al. 2003). However, global three-dimensional multi-fluid calculations are beyond current computational resources. Furthermore, because of dust settling, the dust disk will be much thinner than the gas 
disk, depending on the particle size. The two-dimensional approach will therefore cause fewer discrepancies in the dust distribution. Still, one has to be very careful when interpreting results for planets with $M<0.1 M_{\mathrm{J}}$.

\section{Initial and boundary conditions}

The standard numerical resolution we use is $\left(n_{r}, n_{\phi}\right)=$ $(128,384)$. This way, the cells close to the planet are of equal size in the radial and in the azimuthal directions. We do not resolve the Roche lobe using a low resolution like this, but this resolution is sufficient to capture the long-term global evolution of the disk. For the runs with dust accretion, we used 4 levels of AMR to achieve a resolution that is 16 times higher near the planet.

As mentioned before, we use an aspect ratio of $h=0.05$, and the initial gas surface density distribution is constant, with $\Sigma_{\mathrm{g}}=34 \mathrm{~g} \mathrm{~cm}^{-2}$. This surface density is appropriate for a disk with $0.01 M_{\odot}$ within $100 \mathrm{AU}$ at approximately $7 \mathrm{AU}$, between the location of Jupiter and Saturn in the solar system, or at $13 \mathrm{AU}$ in the Minimum Mass Solar Nebula. Note, however, that the fundamental parameter is the stopping time, and our results can therefore be scaled to disks of arbitrary mass by adopting a different grain size for a given $T_{\mathrm{s}}$.

The initial dust-to-gas ratio is 0.01 . Our distance unit is the radius of the planet's orbit, and in this unit the inner boundary is at $r=0.4$ and the outer boundary at $r=2.5$.

It is easy to see that for a constant surface density the stopping time is also constant:

$T_{\mathrm{s}}=0.0461\left(\frac{\mathrm{s}}{\mathrm{cm}}\right)$,

where the size of the particles $s$ is given in $\mathrm{cm}$.

The gas rotates with a slightly sub-Keplerian velocity due to the radial pressure gradient (note that even when there is no density gradient, there is a pressure gradient caused by the gradient in the sound speed). The dust rotates exactly with the Keplerian velocity, initially. All radial velocities are taken to be zero.

The constant kinematic viscosity is set so that $\alpha=0.004$ at $r=1$ in all models. For the disk described above, the critical planet mass for gap opening is about 1 Jupiter mass $\left(1 M_{\mathrm{J}}\right)$ (Bryden et al. 1999). We varied the mass of the planet between $0.001 M_{\mathrm{J}}$ and $1.0 \mathrm{M}_{\mathrm{J}}$.

The boundary conditions are the same as in Paardekooper \& Mellema (2005), deriving from Godon (1996). They are specifically designed to be non-reflecting, so that all waves generated in the simulated region leave the computational domain without influencing the interior.

\section{Results}

\subsection{Axisymmetric disk}

To begin with, we consider a disk without a planet. The analytic solution for the drift velocity is given in Eq. (15). Figure 3 shows this analytic solution for particles of $1 \mathrm{~mm}$, as well as the numerical solution after 10 orbits of the gas at $r=1$. The analytical solution is indistinguishable from the numerical result, showing that the method handles the large source terms arising due to the small $T_{\mathrm{s}}$ very well. The angular velocity of the dust is always equal to the angular velocity of the gas. The Coriolis force starts to play a role only when the relative radial velocity becomes very large.

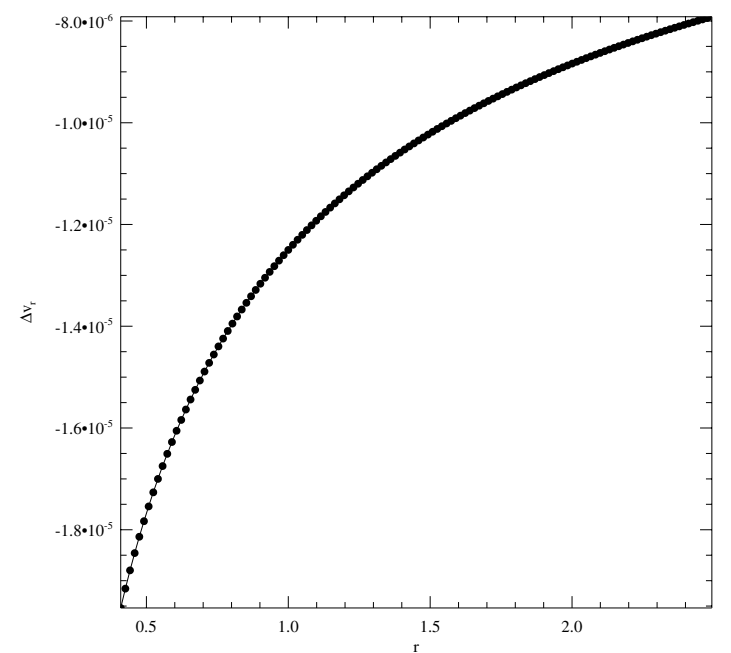

Fig. 3. Relative radial velocity of gas and dust $(1 \mathrm{~mm})$ in a disk without a planet. The dots represent the numerical solution, and the solid line represents Eq. (15).
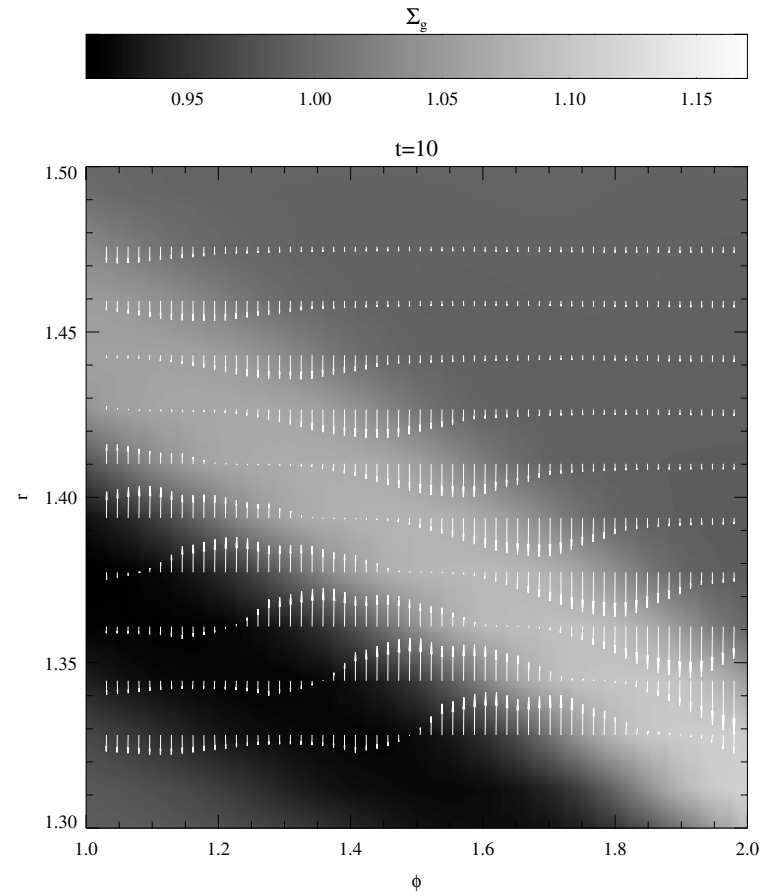

Fig. 4. Close-up on a spiral density wave, with relative velocity arrows superimposed. Outside of the spiral wave, the dust drift is directed inward, according to Eq. (15). Close to the spiral, the particles move towards the centre of the wave. For $5 \mathrm{~mm}$ dust particles, as shown here, the maximum relative velocity is $2.6 \times 10^{-6}$, in units of the orbital velocity of the planet.

\subsection{Dust response to a spiral wave}

An embedded planet perturbs the gas disk by launching spiral density waves. In this section, we investigate the dust response to such a single wave. In Fig. 4, we show a close-up on part of the spiral pattern generated by the planet. The velocity arrows indicate the relative velocity of gas and dust. In the upper right direction, the density and velocity of the gas are close to their initial values, and in this region, the dust drifts slowly inward, according to Eq. (15). In the lower left part of the figure the dust particles drift inward again, but at a higher speed, due to the 


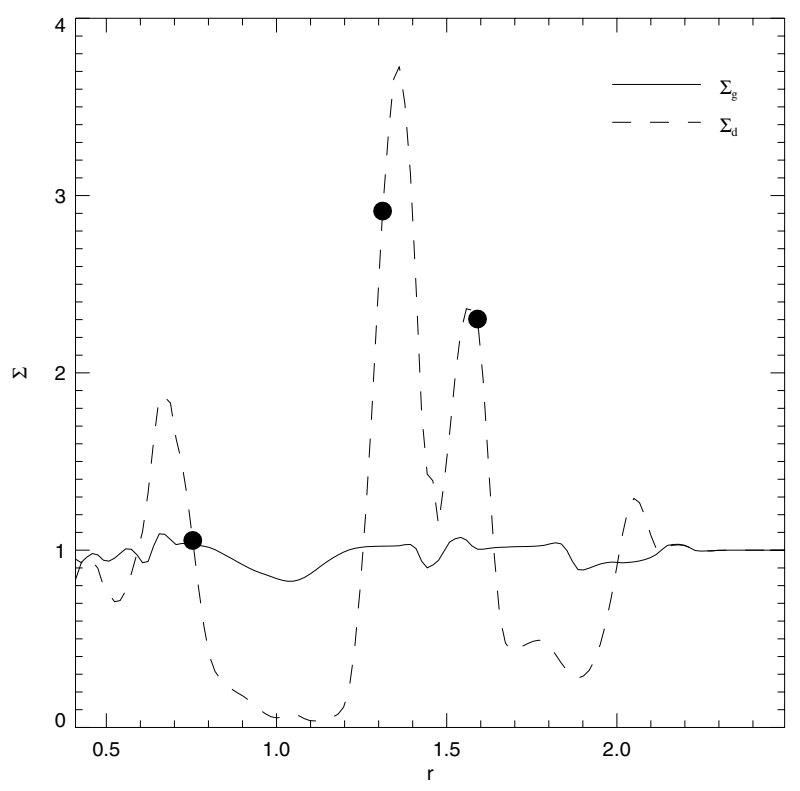

Fig. 5. Surface density of gas and dust $(s=0.1 \mathrm{~cm})$ after 500 orbits of a $0.1 M_{\mathrm{J}}$ planet at $\phi=0$ (opposite to the planet). The dust density is multiplied by 100 . The dots indicate the positions of the $2: 3$, the $3: 2$, and the $2: 1$ resonances.

lower gas density. At the location of the spiral wave, gas density and velocity are such that the dust always moves towards the centre of the wave. This is not surprising in view of Eq. (16), which states that in an axisymmetric disk, dust particles will always move towards the highest pressure. Note that even in this perturbed disk, the angular velocities of gas and dust are still almost equal: the particles move only radially through the gas. Therefore, the dust response to a spiral wave is the same as the response to an axisymmetric density wave, but stretched in the radial direction. The effect of a density wave, as shown in Fig. 4, remains very local and is of minor importance for the global disk evolution.

\subsection{Global disk evolution}

Paardekooper \& Mellema (2004) showed that planets of $\sim 0.1 M_{\mathrm{J}}$ are able to decouple the evolution of the gas disk and the dust disk. More specifically, they showed that the dust particles tend to move away from the orbit of the planet, creating a deep annular gap. Furthermore, the final dust disk appeared to be structured near mean motion resonances. In this section, we further explain the mechanism for structuring the disk.

First of all, Fig. 5 shows a radial density cut through a disk perturbed by a $0.1 M_{\mathrm{J}}$ planet after 500 orbits. Again, as in Paardekooper \& Mellema (2004), we see a clear gap and a density enhancement at the position of the 2:1 mean motion resonance.

Note that since $T_{\mathrm{s}} \ll 1$, the dust particles are never influenced by the planet directly at the radii of the indicated resonances, they only react to the gas density and velocity. The mechanism for structuring the dust disk is therefore intrinsically different from ordinary resonance capture, which is the usual explanation for the existence of the Plutinos in the Kuiper belt (Luu \& Jewitt 2002).

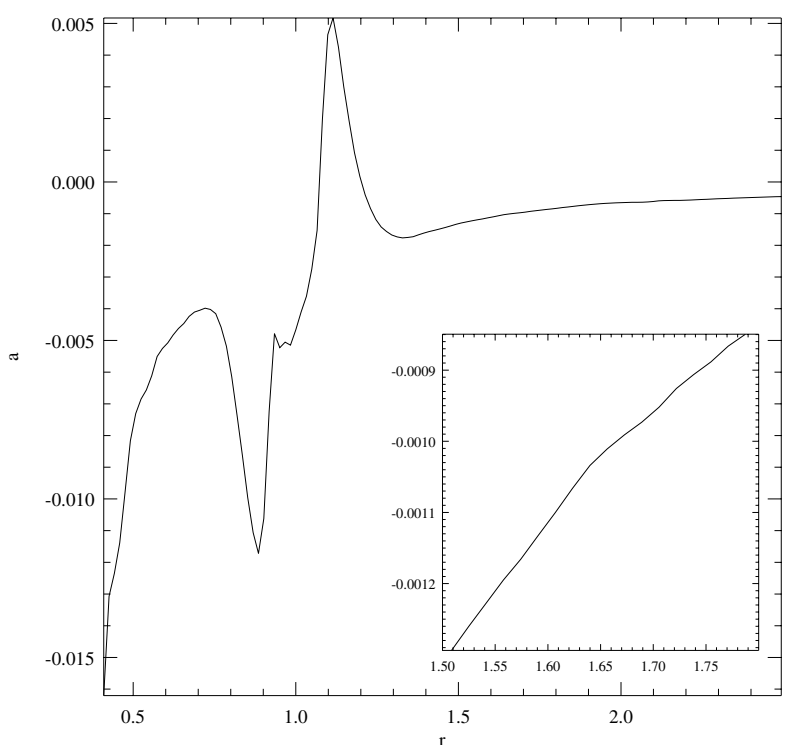

Fig. 6. Azimuthally averaged radial acceleration $a$ of dust particles after 100 orbits of a $0.1 M_{\mathrm{J}}$ planet. The close-up shows $a$ in the outer region of the disk. The 2:1 mean motion resonance is visible as a small bump in $a$.

In Fig. 6, we plot the radial acceleration of dust particles, excluding the drag force:

$a=r\left(v_{\phi}+\Omega\right)^{2}-\frac{\partial \Phi}{\partial r}$.

Figure 6 shows the azimuthally averaged $a$, with a close-up on the outer region of the disk. The first thing to note is that the dust is always accelerated inward, except for the region around $r=1.1$. Inside the planet's orbit, the acceleration is very negative, indicating that the dust will quickly move toward the inner boundary.

The large inward and outward accelerations near $r=0.9$ and $r=1.1$, respectively, are due to the inner and outer edges of the density dip carved in the gas. The pressure gradient at the outer edge is large enough to push the dust outward. The densest ring outside the planet's orbit forms where $a$ switches sign at $r=1.3$. Particles approach this location in the disk from both smaller and larger radii, which leads to a strong dust accumulation.

The close-up in Fig. 6 indicates the outer 2:1 mean motion resonance, which shows up as a bump in the radial acceleration at $r=1.6$. Note that $a$ is still negative near the resonance, so the dust keeps moving inward. However, near the resonance, the dust particles are decelerated, and therefore the dust piles up at that location. From Fig. 5, we can also see that the density peaks just outside the gap are close to, but not exactly located at the position of the 3:2 and the 2:3 resonance, showing that the mechanism by which they are formed is different. In contrast to the region close to the shallow gas gap, there is no apparent pressure gradient near the 2:1 resonance to structure the dust disk. Nonetheless, the resonance does show up in the acceleration in Fig. 6, and it is therefore the velocity field rather than the density of the gas that makes the dust move into the $2: 1$ resonance.

The adopted initial gas surface density is correct for a distance of $13 \mathrm{AU}$ in the Minimum Mass Solar Nebula (MMSN). For this location of the planet, the initial dust mass between $r=1.2$ and $r=1.5$ is 5.4 Earth Masses $\left(M_{\oplus}\right)$. After 500 orbits of the planet, the dust mass in this region has grown to $10.9 M_{\oplus}$, an enhancement of more than a factor of 2 (see Fig. 5). This may 
have serious implications for building planetesimals (boulders with sizes larger than 10 centimeters) in this region.

We have not included dust diffusion in our models. Turbulent transport of small dust grains is not yet fully understood, but it can be described by diffusion for the smallest particles (Johansen $\&$ Klahr 2005). To see what effect diffusion has on dust-gap formation, we gave the dust fluid the same turbulent diffusion coefficient as the gas, and included the viscous force terms in the dust source update. It turned out that dust diffusion affects the dust density distribution only at the $1 \%$ level, indicating that this is not an important process. However, the largest particles we consider may be subject to vortex trapping in turbulent eddies (Johansen \& Klahr 2005), and for these particles, the diffusion approach may not be entirely valid.

\subsection{Dependence on particle size}

We have seen that particles of $1 \mathrm{~mm}$ decouple from the gas due to the disk perturbation induced by a small planet. As we examine smaller and smaller particles, we expect a certain minimum particle size for this decoupling to occur because smaller particles couple better to the gas. We can make a simple estimate for this $s_{\min }$ by looking at the region in the disk near $r=1.1$, where the dust experiences the largest positive acceleration $a_{\max }$. According to Fig. $6, a_{\max }=0.005$. The radial gas velocity in a standard accretion disk is

$v_{r, \mathrm{~g}}=-\frac{3 v}{2 r}$

The gas radial velocity in a disk with a planet is similar. Balancing the outward acceleration with the radial drag force gives an equilibrium velocity difference between gas and dust of

$\Delta v_{r}=a_{\mathrm{max}} \frac{T_{\mathrm{s}}}{\Omega_{\mathrm{K}}}$.

When this velocity difference is larger than the radial gas velocity, dust particles can be accelerated outward around $r=1.1$, creating a low density gap and a high density ring. The minimum stopping time for this to occur is

$T_{\mathrm{s}, \min }=\frac{3 \Omega_{\mathrm{K}} v}{2 r a_{\max }}$.

For our disk parameters, this implies a minimum particle size of approximately $500 \mu \mathrm{m}$. Note, however, that although smaller particles will not be pushed outward, they will travel inward at a different speed near the edge of the gas dip. As in the case for the 2:1 resonance, where in spite of the acceleration being negative everywhere a dust ring still arises, these particles will eventually create a gap, but on a much longer time scale.

Figure 7 shows the radial dust density for four different particle sizes after 100 orbits. First of all, it is clear that the larger particles react even more dramatically on the planet. The three bumps at $r=0.7, r=1.3$, and at corotation all grow very fast with increasing particle size. After 400 orbits, the dust-to-gas ratio of $5 \mathrm{~mm}$ particles at $r=1.3$ is enhanced by more than an order of magnitude. The width of the dust gap does not depend on the size of the particles.

The outer disk $(r>1.1)$ reaches a quasi-static state after approximately 100 orbits. From then on the only evolution is in the further growth of existing features. The inner disk is slowly cleared, however, but on a time scale that is set by the unperturbed motion of gas and dust. If we take Eq. (32) for the

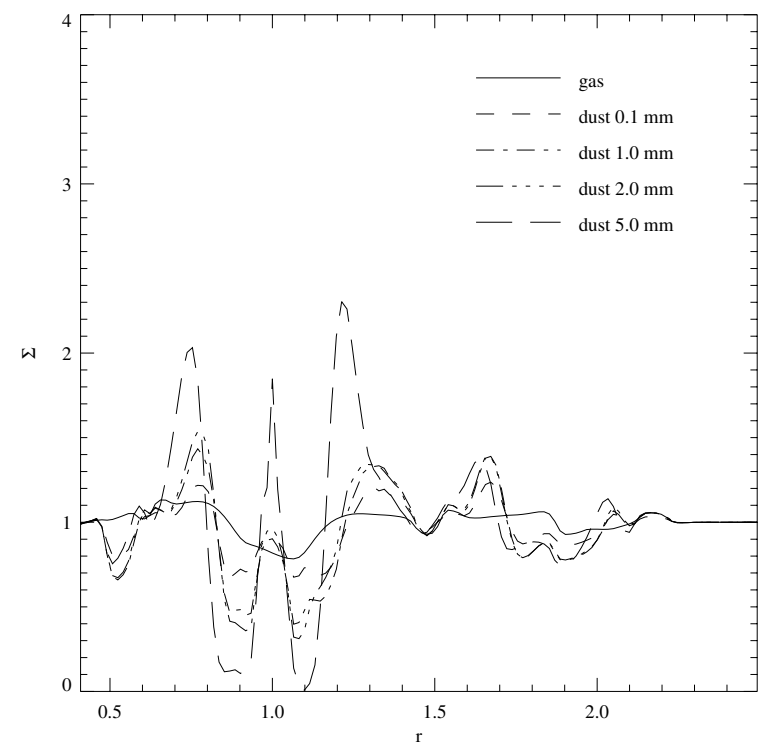

Fig. 7. Radial density cut opposite to the planet for four different particle sizes after 100 orbits of a $0.1 M_{\mathrm{J}}$ planet. The dust densities are multiplied by 100 .

velocity of the gas and Eq. (15) for the relative velocity, we can write the following for the dust velocity:

$v_{r}=-\left(\frac{3}{2} \alpha+T_{\mathrm{s}}\right) h^{2} v_{\mathrm{K}}$

This gives a time scale for clearing the region inside $r=1$ of approximately 50000 orbits.

Looking at the distribution of $0.1 \mathrm{~mm}$ particles in Fig. 7, we see that even these well-coupled particles start to create a clearing in the inner disk. However, since this planet is not strong enough to keep all the dust outside of $r=1.3$, the inner disk is still fed from outside. Particles larger than $500 \mu \mathrm{m}$ are able to create a fast gap for this planet. This agrees with the estimate of Eq. (34). Based in Fig. 7, we estimate that particles larger than $150 \mu \mathrm{m}$ will open up a dust gap within 500 orbits.

\subsection{Dependence on planetary mass}

Smaller planets do not dramatically perturb the disk, so we expect that a minimum planetary mass for dust-gap opening also exists. In this section, we consider only $1 \mathrm{~mm}$ particles.

Figure 8 shows the radial dust distributions for four different planetary masses. The smallest planet is not able to produce a density dip near its orbit, so the gas density is equal to 1 everywhere except in the spiral waves. From Fig. 8, we conclude that a planet of $0.05 M_{\mathrm{J}}$ is the lowest-mass planet that is able to create a gap, albeit on a longer time scale than for more massive planets.

The highest mass planet in Fig. 8 is on the edge of creating a gap in the gas disk. As a result of the lower gas density, the stopping time for particles near the orbit of the planet is relatively large, which allows for the strong evolution near $r=1$. The time scale for dust-gap formation for this planet is about half the time scale for gas-gap formation, which is approximately 100 planetary orbits. Also, due to the strong spiral waves, the 2:1 mean motion resonance plays a major role; the resonance is able to suck material up from both sides, leading to a less pronounced peak near $r=1.4$ and to an empty region around $r=1.8$. Figure 9 shows the dust surface density for this planet. 


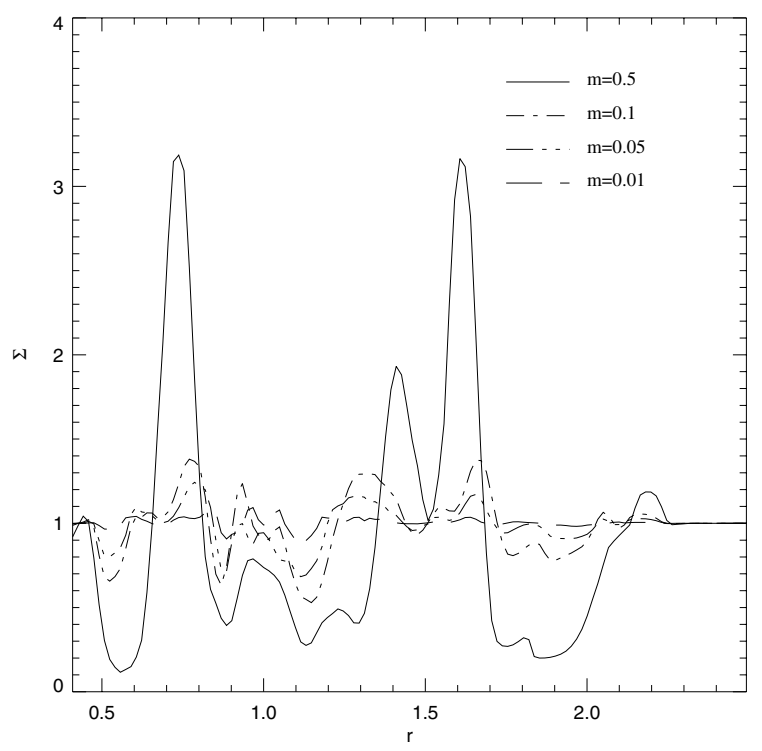

Fig. 8. Radial density cut opposite to the planet for four different planetary masses after 100 orbits and for a particle size of $1 \mathrm{~mm}$. The dust densities are multiplied by 100 , and the planetary masses are in units of $M_{\mathrm{J}}$.
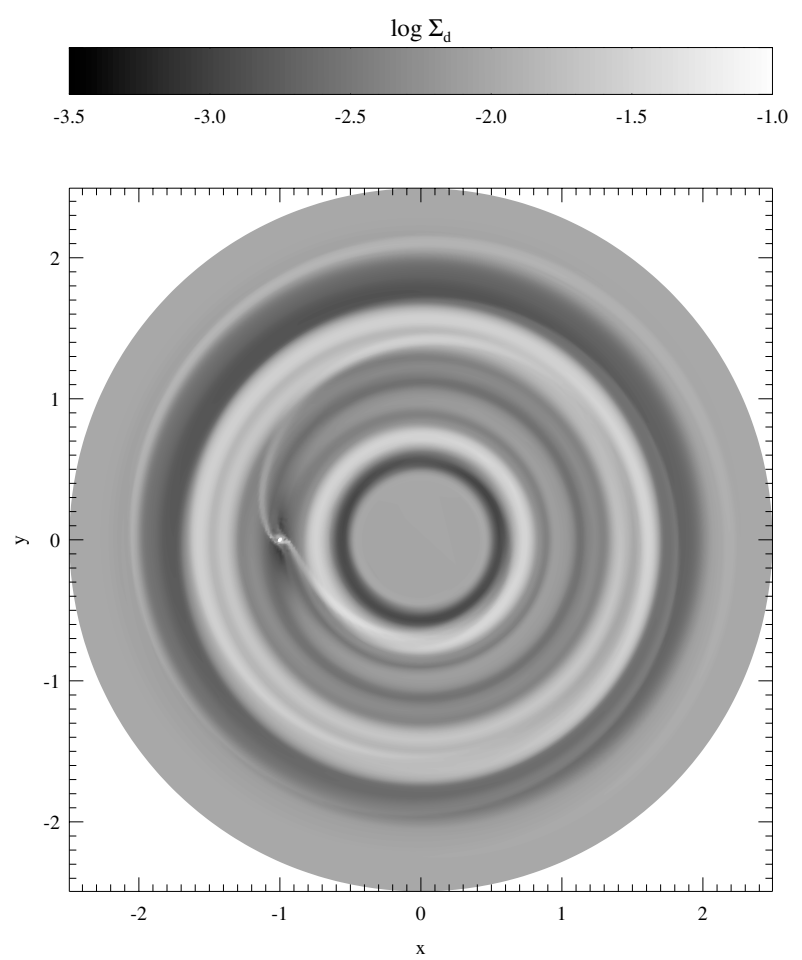

Fig. 9. Surface density of $1 \mathrm{~mm}$ dust particles after 100 orbits of a $0.5 M_{\text {J }}$ planet.

Note that the resonance-induced gaps at $r=0.55$ and $r=1.8$ are as deep as the gap near the planet's orbit. Observationally, it may be difficult to distinguish a single-planet system like this from one with multiple planets.

Because of the conditions discussed in Sect. 3.5, a $0.5 M_{\mathrm{J}}$ planet is the maximum planetary mass we can accurately model with a two-fluid calculation. However, based on the relation between the radial acceleration $a$ and the resulting dust distribution (compare Figs. 5 and 6), we can still make a few remarks concerning higher-mass planets.

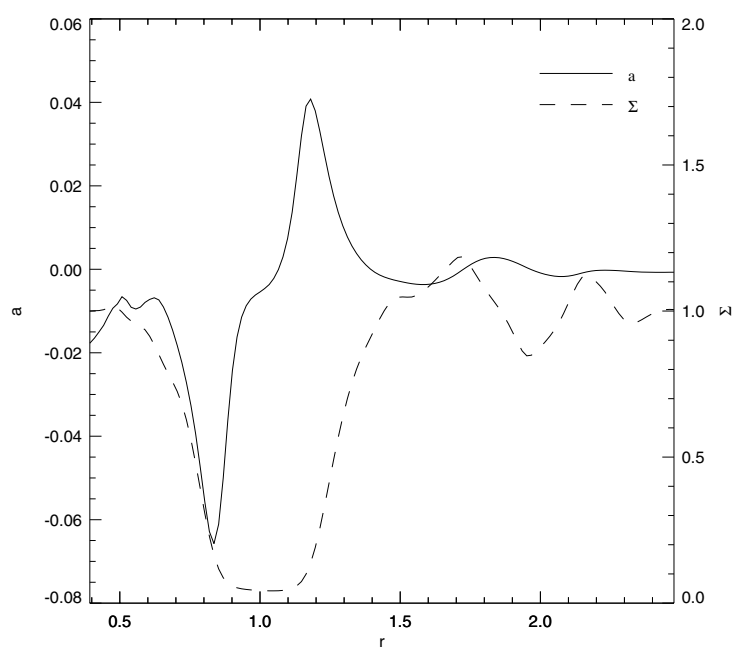

Fig. 10. Azimuthal averages of the radial acceleration $a$ (solid line) and gas surface density $\Sigma$ (dashed line, shown for $0.4 \leq r \leq 1.8$ ) for a $1 M_{\mathrm{J}}$ planet after 400 orbits.

First of all, we expect similar things to happen for a $1 M_{\mathrm{J}}$ planet as for a $0.5 M_{\mathrm{J}}$ planet, only in a more extreme form. Secondly, we noted in Sect. 5.3 that the dust ring just outside the gap forms where $a$ changes sign from positive to negative. In Fig. 10, we show $a$ together with the gas surface density $\Sigma$ for a $1 M_{\mathrm{J}}$ planet. The gas gap clearly stands out, and we see that $a$ changes sign at the outer edge of the gap around $r=1.4$. A dust ring at that location would coincide with the outer edge of the gas gap. Therefore, a possible dust gap would have the same width as the gap in the gas disk.

Also from Fig. 10, we see that $a$ is structured up to $r \approx 2.5$, which is larger than for the $0.1 M_{\mathrm{J}}$ planet (see Fig. 6). For a planet at Neptune's orbit (semi-major axis $30 \mathrm{AU}$ ), the resonant structure in the dust disk can therefore extend to at least $75 \mathrm{AU}$.

\subsection{Flow within the Roche lobe}

The flow of dust deep within the Roche lobe $\left(R_{\mathrm{R}}\right)$ of the planet is relatively simple. Due to the strong pressure gradient in the direction of the planet, the relative velocity of the dust particles is always directed towards the planet. In other words, if we take a cylindrical coordinate frame $(s, \theta)$ centred on the planet, then $\Delta v_{s}=v_{s, d}-v_{s, g}$ is always negative.

In Fig. 11, we show this relative velocity as a function of distance to a $0.1 M_{\mathrm{J}}$ planet for $1 \mathrm{~mm}$ particles. The first thing to note is that the relative velocity changes sign very close to the planet. That is because this was a run with gas and dust accretion, and therefore most of the gas inside $0.1 R_{\mathrm{R}}$ was removed (see Paardekooper \& Mellema 2005, for the accretion procedure), leading to a reversed pressure gradient locally. Outside the accretion area, dust moves slowly towards the planet with respect to the gas. The spread in velocities stems from the fact that the gas density distribution around the planet is not symmetric with respect to the planet (D'Angelo et al. 2002).

If we take the average relative velocity in the Roche lobe as $4.0 \times 10^{-4}$ in units of the Kepler velocity at the location of the planet, then we get a time scale to clear the Roche lobe of dust of less then 100 orbits for particles of $1 \mathrm{~mm}$. This is an important time scale for accretion, as we will see in the next section. 


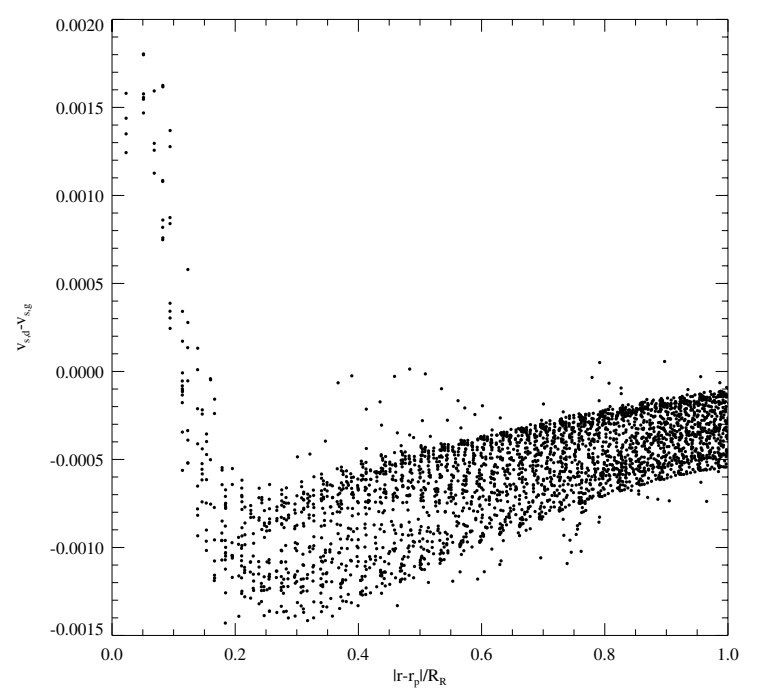

Fig. 11. Scatter plot of the relative velocity in the direction of a $0.1 M_{\mathrm{J}}$ planet. The distance to the planet is in units of the Roche lobe $R_{\mathrm{R}}$, and the unit of relative velocity is the Kepler velocity at the position of the planet. The size of the dust particles in this simulation is $2 \mathrm{~mm}$.

\subsection{Gas and dust accretion}

We now turn to the interesting problem of dust accretion. The accretion of dust particles compared to the gas is important for the final enrichment of gaseous giant planets. When the dust accretion rate is higher than 0.01 (the initial dust-to-gas ratio) times the gas accretion, the planet will get enriched in solids, and when the dust accretion is lower than 0.01 the gas-dust mixture that is accreted onto the planet is relatively gas-rich. In the discussion below, we multiply the dust content of the disk by 100 , so that gas and dust accretion can be directly compared.

The mass range we can consider is limited because for $M_{p}<0.1 M_{\mathrm{J}}$, three-dimensional effects start to play an important role (D'Angelo et al. 2003), while planets larger than approximately $0.5 M_{\mathrm{J}}$ open up a deep gas gap in the disk, which we cannot treat in the fluid approach. Therefore, to eludicate the basic principles that govern dust accretion and to provide a starting point for future, more detailed simulations, we only consider a $0.1 M_{\text {J }}$ planet.

In the left panel of Fig. 12, we show the accreted mass of gas and dust in units of gas and dust disk masses as a function of time. The accretion of gas (solid line) was extensively discussed in Paardekooper \& Mellema (2005) and is shown here only for comparison. After approximately 200 orbits, it reaches a constant value of $1.5 \times 10^{-4}$ gas disk masses per orbit.

The accretion rates for dust particles of different sizes show a different behaviour. In the first few orbits, relatively more dust is accreted than gas, which means that the dust mass that is accreted is larger than 0.01 times the gas mass that is accreted. This is the stage where the immediate surroundings of the Roche lobe are cleared of dust, due to the strong pressure gradients in the planetary atmosphere. Later, the competing process of dust-gap formation sets in, and consequently, dust accretion slows down. In the end, for all particle sizes, relatively more gas is accreted than dust, which means that the planet will not be enriched in solids of these particular sizes.

The right panel of Fig. 12 shows the ratio of accretion rates for the different particle sizes to the gas accretion rate. We see that initially the accretion rate of dust is higher than 0.01 times the accretion rate of the gas or all particle sizes, corresponding to the Roche lobe clearing mentioned before. If we look at the accretion rate of $1 \mathrm{~mm}$ particles, after the first sharp peak, the accretion rate stays almost constant for 30 orbits, after which the process of gap formation sets in, and the accretion rate slowly declines. After 300 orbits, the accretion rate for the dust is less than half the accretion rate of the gas, and it is still declining.

For the larger particles, we see a larger initial accretion rate because these more weakly coupled dust particles move up the strong pressure gradient near the planet more easily. However, for larger particles, gap formation sets in earlier, as well, and it will eventually win. A particle size of $2 \mathrm{~mm}$ results in the largest accreted mass of dust in this first stage of accretion. For $4 \mathrm{~mm}$ particles, gap formation sets in before the planet can accrete a significant amount of surrounding dust. However, in the long run, more small than large particles will have been accreted, as can be seen from the left panel of Fig. 12.

The planet clears the outer part of the gap of $4 \mathrm{~mm}$ particles in approximately 100 orbits (see Fig. 7). At this point, the accretion rate curve levels off at a value of $0.2 \dot{M}_{\text {gas }}$, but around 150 orbits, it starts to decrease again. Accretion around this time comes from the dense ring at $r=1$, which will slowly diffuse into the inner disk. After it is gone, dust accretion will be negligible compared to gas accretion.

\section{Discussion}

Dust gaps opened by low-mass planets have some interesting observational and theoretical consequences. In Paardekooper \& Mellema (2004), it was shown that the Atacama Large Millimeter Array (ALMA) will provide the resolution to observe these gaps in protoplanetary disks at the distance of the Taurus starforming region. Observationally, the relatively large particles we consider are only important at $\mathrm{mm}$-wavelengths. To create gaps and inner holes in disks that can be detected at infrared wavelengths, more massive planets are needed to remove the smallest particles.

Next, we will explore some more possible consequences for planet formation. Before we can make any quantitative statements about the importance of the limited size range we consider in this paper, we need to determine what fraction of the total dust mass these particles represent. If we look at the size distribution from Fig. 7 of Dullemond \& Dominik (2005) we see a bimodal distribution (dust and planetesimals), with a transition around particles of $10 \mathrm{~cm}$. It is interesting to note that for typical mid-plane densities, a size of $10 \mathrm{~cm}$ gives rise to $T_{\mathrm{s}} \approx 1$ (see Eq. (30)), which is the upper limit for which the fluid approach is valid. Boulders larger than $10 \mathrm{~cm}$ are not influenced by the gas as much as the smaller particles, and therefore will not participate in gap formation due to pressure gradients, while particles smaller than $150 \mu \mathrm{m}$ are too well-coupled to the gas to form a gap (see Sect. 5.4). From Fig. 7 of Dullemond \& Dominik (2005), we estimate that half of the total mass of particles smaller than $10 \mathrm{~cm}$ is in particles larger than $150 \mu \mathrm{m}$. Furthermore, about $30 \%$ of the total dust mass is in particles with sizes between $150 \mu \mathrm{m}$ and $10 \mathrm{~cm}$.

The above estimate implies that the inner disk is denied $30 \%$ of the amount of solids it would receive from the outer disk without the presence of the planet. If (terrestrial) planets are still being formed in the inner disk, it may slow down this process considerably.

Dust piles up outside the planet's orbit the (see Fig. 5). In Sect. 5.3, we mentioned that after 500 orbits of the planet, the amount of dust had already doubled. Because the planet acts as a barrier for particles moving inward, more and more dust will 


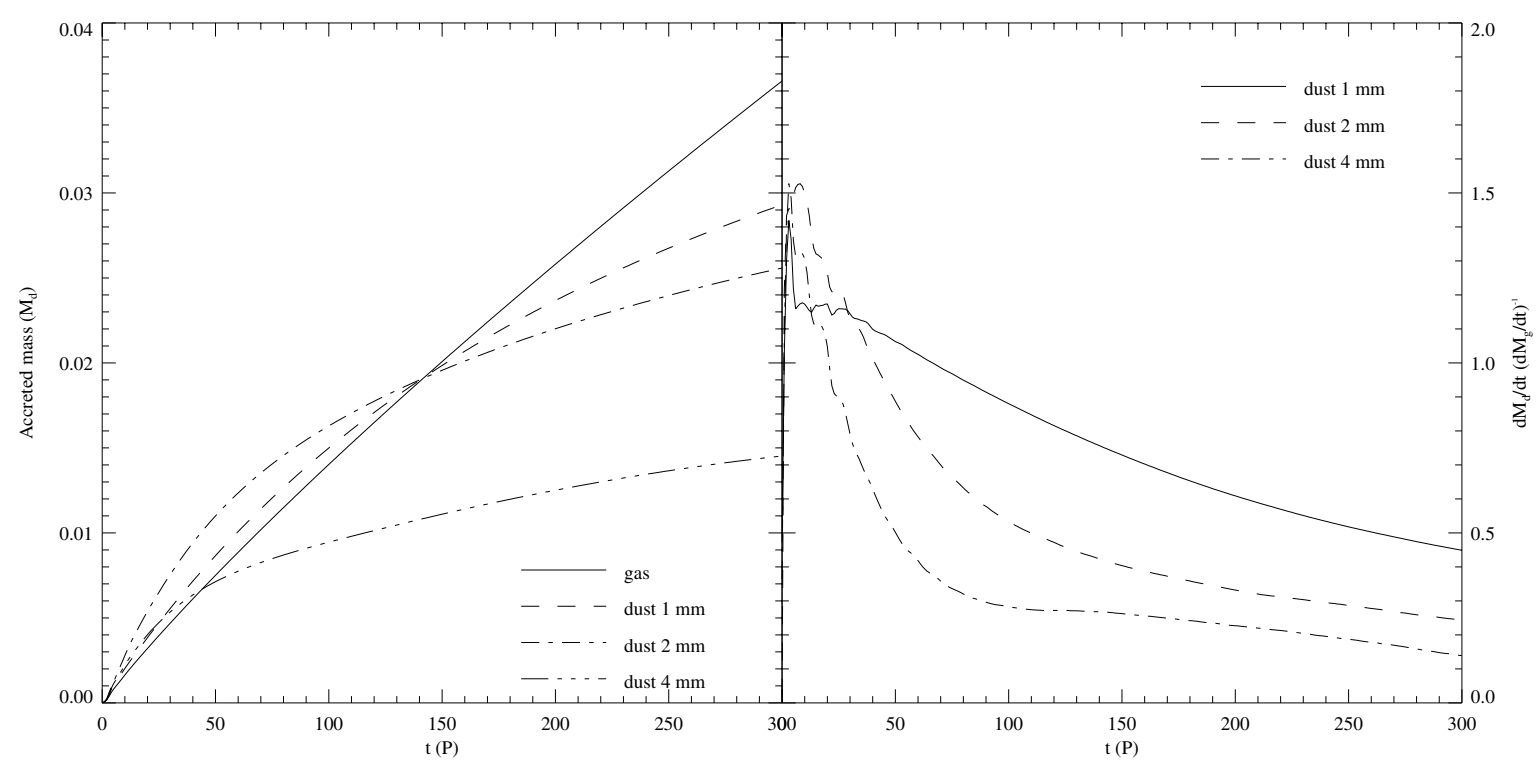

Fig. 12. Accretion of gas and dust onto a $0.1 M_{\mathrm{J}}$ planet. Left panel: gas and dust mass accreted on the planet. Right panel: relative accretion rate of dust with respect to the gas. For both panels, the dust mass is multiplied by 100 to compare it with the gas.

stream into the resonance from the outer disk. It is interesting to note that this is in the region where the Kuiper Belt resides with respect to Neptune. This large amount of dust may well have influenced planetesimal formation in that region.

If an already-formed giant planet is present in the inner disk, this planet is also denied $30 \%$ of the amount of solids it would be able to accrete without the presence of the outer planet. One could imagine that in the present configuration of our Solar System, with three dust-gap-opening planets outside 5.2 AU, the amount of solid material accreted by Jupiter would be dramatically small, even without the mechanism outlined in Sect. 5.7.

Depending on the dust size distribution in the disk, the low relative accretion rates for $\mathrm{mm}$-sized particles found in Sect. 5.7 will have a significant effect on the final composition of the planet. Our results show that the accretion rates for these particles are, on the long run, more than an order of magnitude lower than the gas accretion rate. This will significantly affect the final enrichment in solids for giant planets, even more so if a dustgap-opening planet exists in the outer disk.

In other words, if, as is standard in planet-formation models, one considers gas accretion and the accretion of planetesimals separately, the gas that is accreted is relatively poor in solids (by a factor of 2) compared to the solar value. This process works against the usual enrichment scenarios for the giant planets (Gautier et al. 2001a,b; Owen et al. 1999). However, as was mentioned before, we cannot make firm statements about planets of higher mass. More definitive conclusions about dust accretion onto giant planets require further study.

Dust growth may also play an important role in structuring the disk. When the growth time scale $\tau_{\mathrm{g}}$ is long compared to the dust-gap-formation time scale, what effectively happens is that particles will be removed from the dust gap as soon as they become large enough. This means that the gap will also be cleared of the smallest particles on a time scale $\tau_{\mathrm{g}}$. However, the process of dust growth in disks is not understood well enough to make any quantitative statements about it.

It is important to realize that the dust-gap-formation process basically only needs a shallow gas dip formed by an embedded planet. As long as more complicated disk models including magnetic fields and radiative transfer do not change this gas structure, the basic picture outlined in this paper will not change. Three-dimensional effects may be important, however, because gas and dust behave differently in the vertical direction: dust particles will settle to the midplane, while the gas forms a pressure-supported structure. Angular momentum transport, and therefore also gap formation, is reduced in three-dimensional models, compared to vertically integrated models, but because of settling effects, the dust will mainly interact with gas near the midplane where the planetary potential is strongest. It is not clear how these competing processes will affect our results; threedimensional, multi-fluid simulations are needed to answer that question.

\section{Summary and conclusions}

In this paper, we have elaborated on the findings of Paardekooper \& Mellema (2004), who showed that intermediate mass planets are able to open dust gaps in gas disks. We have shown how the formation of the gap depends on particle size and planetary mass, indicating that particles larger than $150 \mu \mathrm{m}$ and planets larger than $0.05 M_{\mathrm{J}}$ are needed to open a gap in a typical protoplanetary disk. Planets larger than $0.5 M_{\mathrm{J}}$ clear a gas gap around their orbit, increasing the stopping time $T_{\mathrm{s}}$ locally by 2 orders of magnitude, leaving the dust inside the gap largely decoupled from the gas. If the planet clears a gas gap, the dust gap will be of equal width.

Within the Roche lobe of the planet, the pressure gradient is such that solids will quickly move to the centre of the planetary atmosphere, allowing for a very high dust accretion rate. However, this enhancement is more than counterbalanced by the local depletion of dust due to the formation of the dust gap. In the end, much more gas can be accreted compared to solids. This may have serious consequences for the enrichment of giant planets.

Acknowledgements. S.P. and G.M. acknowledge financial support from the European Research Training Network "The Origin of Planetary Systems" (PLANETS, contract number HPRN-CT-2002-00308) at Leiden Observatory. G.M.'s work in Leiden is made possible through support from the Royal Netherlands Academy of Arts and Sciences. 


\section{References}

Boss, A. P. 1997, Science, 276, 1836

Bryden, G., Chen, X., Lin, D. N. C., Nelson, R. P., \& Papaloizou, J. C. B. 1999 , ApJ, 514, 344

D’Angelo, G., Henning, T., \& Kley, W. 2002, A\&A, 385, 647

D'Angelo, G., Kley, W., \& Henning, T. 2003, ApJ, 586, 540

Dullemond, C. P., \& Dominik, C. 2005, A\&A, 434, 971

Eulderink, F., \& Mellema, G. 1995, A\&AS, 110, 587

Fromang, S., \& Nelson, R. P. 2005, MNRAS, 364, L81

Garaud, P., Barrière-Fouchet, L., \& Lin, D. N. C. 2004, ApJ, 603, 292

Gautier, D., Hersant, F., Mousis, O., \& Lunine, J. I. 2001a, ApJ, 550, L227

Gautier, D., Hersant, F., Mousis, O., \& Lunine, J. I. 2001b, ApJ, 559, L183

Godon, P. 1996, MNRAS, 282, 1107

Goldreich, P., \& Ward, W. R. 1973, ApJ, 183, 1051

Guillot, T., Stevenson, D. J., Hubbard, W. B., \& Saumon, D. 2004, The interior of Jupiter (Jupiter. The Planet, Satellites and Magnetosphere), 35

Haghighipour, N., \& Boss, A. P. 2003, ApJ, 598, 1301

Johansen, A., Henning, T., \& Klahr, H. 2005, ArXiv Astrophysics e-prints Johansen, A., \& Klahr, H. 2005, ApJ, 634, 1353

Johansen, A., Klahr, H., \& Henning, T. 2006, ApJ, 636, 1121

Kley, W. 1999, MNRAS, 303, 696
Kwok, S. 1975, ApJ, 198, 583

Leveque, R. J. 2004, Journal of Hyperbolic Differential Equations, 1, 315

Lubow, S. H., Seibert, M., \& Artymowicz, P. 1999, ApJ, 526, 1001

Luu, J. X., \& Jewitt, D. C. 2002, ARA\&A, 40, 63

Nelson, R. P., Papaloizou, J. C. B., Masset, F., \& Kley, W. 2000, MNRAS, 318, 18

Owen, T., Mahaffy, P., Niemann, H. B., et al. 1999, Nature, 402, 269

Paardekooper, S.-J., \& Mellema, G. 2004, A\&A, 425, L9

Paardekooper, S.-J., \& Mellema, G. 2005, A\&A, in press

Pollack, J. B., Hubickyj, O., Bodenheimer, P., et al. 1996, Icarus, 124, 62

Roe, P. L. 1981, J. Comp. Phys, 43, 357

Shakura, N. I., \& Sunyaev, R. A. 1973, A\&A, 24, 337

Simis, Y. J. W., Icke, V., \& Dominik, C. 2001, A\&A, 371, 205

Sod, G. A. 1978, J. Comp. Phys, 27, 1

Suttner, G., \& Yorke, H. W. 2001, ApJ, 551, 461

Takeuchi, T., \& Artymowicz, P. 2001, ApJ, 557, 990

Takeuchi, T. \& Lin, D. N. C. 2002, ApJ, 581, 1344

Weidenschilling, S. J. 1977, MNRAS, 180, 57

Woitke, P., \& Helling, C. 2003, A\&A, 399, 297

Wolf, S., Gueth, F., Henning, T., \& Kley, W. 2002, ApJ, 566, L97

Woodward, P., \& Colella, P. 1984, Journal of Computational Physics, 54, 115

Youdin, A. N., \& Goodman, J. 2005, ApJ, 620, 459 